\title{
Towards Surface Realization with CCGs Induced from Dependencies
}

\author{
Michael White \\ Department of Linguistics \\ The Ohio State University \\ Columbus, OH 43210, USA \\ mwhitedling.osu.edu
}

\begin{abstract}
We present a novel algorithm for inducing Combinatory Categorial Grammars from dependency treebanks, along with initial experiments showing that it can be used to achieve competitive realization results using an enhanced version of the surface realization shared task data.
\end{abstract}

\section{Introduction}

In the first surface realization shared task (Belz et al., 2011), no grammar-based systems achieved competitive results, as input conversion turned out to be more difficult than anticipated. Since then, Narayan \& Gardent (2012) have shown that grammar-based systems can be substantially improved with error mining techniques. In this paper, inspired by recent work on converting dependency treebanks (Ambati et al., 2013) and semantic parsing (Kwiatkowksi et al., 2010; Artzi and Zettlemoyer, 2013) with Combinatory Categorial Grammar (CCG), we pursue the alternative strategy of inducing a CCG from an enhanced version of the shared task dependencies, with initial experiments showing even better results.

A silver lining of the failure of grammar-based systems in the shared task is that it revealed some problems with the data. In particular, it became evident that in cases where a constituent is annotated with multiple roles in the Penn Treebank (PTB), the partial nature of Propbank annotation and the restriction to syntactic dependency trees meant that information was lost between the surface and deep representations, leading grammarbased systems to fail for good reason. For example, Figure 1 shows that with free object relatives, only one of the two roles played by how much manufacturing strength is captured in the deep representation, making it difficult to linearize this phrase correctly. By contrast, Figure 2 (top)

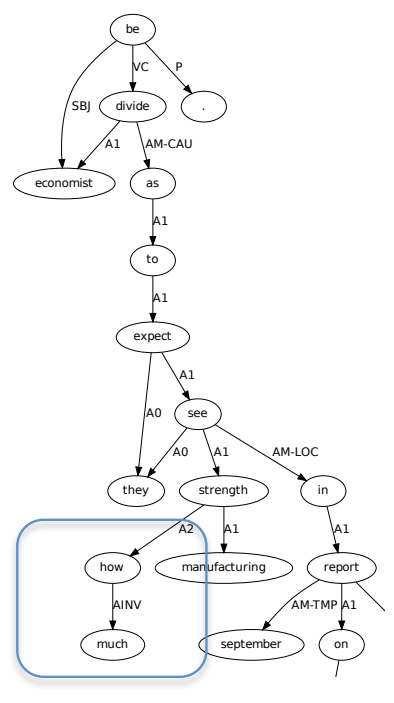

Figure 1: Shared Task Input for Economists are divided as to [how much manufacturing strength $]_{i}$ they expect to see $t_{i}$ in September reports on industrial production and capacity utilization, also due tomorrow (wsj_2400.6, "deep" representation)

shows an experimental version of the shallow representation intended to capture all the syntactic dependencies in the PTB, including the additional object role played by this phrase here. ${ }^{1}$ Including all PTB syntactic dependencies in the shallow representation makes it feasible to define a compatible CCG; at the bottom of the figure, a corresponding CCG derivation for these dependencies is shown. In the next section, we present an algorithm for inducing such derivations. In contrast to Ambati et al.'s (2013) approach, the algorithm integrates the proposal of candidate lexical categories with the derivational process, making it possible to derive categories involving unsaturated arguments, such as $\mathrm{s}_{e, \mathrm{dcl}} \backslash \mathrm{np}_{x} /\left(\mathrm{s}_{e^{\prime}, \text { to }} \backslash \mathrm{np}_{x}\right)$; it also makes greater use of unary type-changing rules, as with Artzi \& Zettlemoyer's (2013) approach.

\footnotetext{
${ }^{1}$ Kudos to Richard Johansson for making these enhancements available.
} 
Unlike their approach though, it works in a broad coverage setting, and makes use of all the combinators standardly used with CCG, including ones for type-raising.

\section{Inducing CCGs from Dependencies}

Pseudocode for the induction algorithm is given in Figure 3. The algorithm takes as input a set of training sentences with their gold standard dependencies. We pre-processed the dependencies to make coordinating conjunctions the head, and to include features for zero-determiners. The algorithm also makes use of a seed lexicon that specifies category projection by part of speech as well as a handful of categories for function words. For example, (1) shows how a tensed verb projects to a finite clause category, while (2) shows the usual CCG category for a determiner, which here introduces a $\langle$ NMOD $\rangle$ dependency. ${ }^{2}$

(1) expect $\vdash \mathrm{s}_{e, \mathrm{dcl}}: @_{e}($ expect $\wedge\langle$ TENSE $\rangle$ pres $)$

(2) the $\vdash \mathrm{np}_{x} / \mathrm{n}_{x}: @_{x}(\langle\mathrm{NMOD}\rangle(d \wedge$ the $))$

The algorithm begins by instantiating the lexical categories and type-changing rules that match the input dependency graph, tracking the categories in a map (edges) from nodes to edges (i.e., signs with a coverage vector). It then recursively visits each node in the primary dependency tree bottom up (combineEdges), using a local chart (doCombos) at each step to combine categories for adjacent phrases in all possible ways. Along the way, it creates new categories (extendCats and coordCats) and unary rules (applyNewUnary). For example, when processing the node for expect in Figure 2, the nodes for they and to are recursively processed first, deriving the categories $n p_{w_{9}}$ and $s_{w_{11}, t o} \backslash n p_{w_{9}} / n p_{w_{8}}$ for they and to see ..., respectively. The initial category for expect is then extended as shown in (3), which allows for composition with to see ... (as well as with a category for simple application). When there are coordination relations for a coordinating conjunction (or coordinating punctuation mark), the appropriate category for combining like types is instead constructed, as in (4). Additionally, for modifiers, unary rules are instantiated and applied, e.g. the rule for noun-noun compounds in (5).

\footnotetext{
${ }^{2}$ In the experiments reported here, we made use of only six (non-trivial) hand-specified categories and two typechanging rules; though we anticipate adding more initial categories to handle some currently problematic cases, the vast majority of the categories in the resulting grammar can be induced automatically.
}

Inputs Training set of sentences with dependencies. Initial lexicon and rules. Argument and modifier relations. Derivation scoring metric. Maximum agenda size.

Definitions edges is a map from dependency graph nodes to their edges, where an edge is a CCG sign together with a coverage bitset; agenda is a priority queue of edges sorted by the scoring metric; chart manages equivalence classes of edges; see text for descriptions of auxiliary functions such as extendCats and coordCats below.

\section{Algorithm}

bestDerivs, lexcats, unaryRules $\leftarrow \emptyset$

For each item in training set:

1. edges[node $] \leftarrow$ instCats (node), ruleInsts $[$ node $] \leftarrow$ instRules(node), for node in input graph

2. combineEdges(root), with root of input graph

3. bestEdge $\leftarrow$ unpack(edges[root $])$; bestDerivs $+\leftarrow$ bestEdge.sign; lexcats $+\leftarrow$ abstractedCats(bestEdge), unaryRules $+\leftarrow$ abstractedRules(bestEdge), if bestEdge complete

def combineEdges(node):

1. combineEdges(child) for child in node.kids

2. edges[node] $+\leftarrow$ coordCats(node) if node has coord relations, otherwise edges[node] $\leftarrow$ extendCats(node,rels) for argument rels

3. agenda $\leftarrow$ edges[node]; agenda $+\leftarrow$ edges[child] for child in node.kids; chart $\leftarrow \emptyset$

4. While agenda not empty:

(a) next $\leftarrow$ agenda.pop

(b) chart $+\leftarrow$ next

(c) doCombos(next), unless next packed into an existing chart item

5. edges[node] $\leftarrow$ chart edges for node filtered for maximal input coverage

def doCombos(next):

1. agenda $+\leftarrow$ applyUnary(next), if next is for node

2. For item in chart:

(a) agenda $+\leftarrow$ applyBinary(next,item), if next is adjacent to item

(b) agenda $+\leftarrow$ applyNewUnary(next,item), if next connected to item by a modifier relation

Outputs bestDerivs, lexcats, unaryRules

Figure 3: CCG Induction Algorithm 


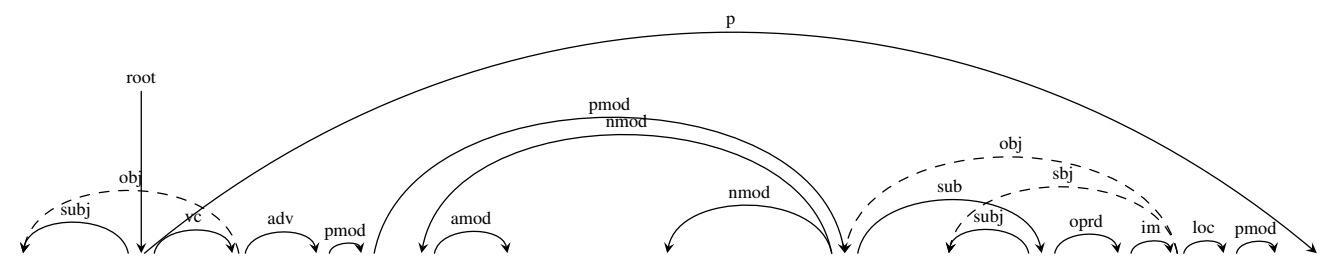

economists are divided as to how much manufacturing strength they expect to see in ....

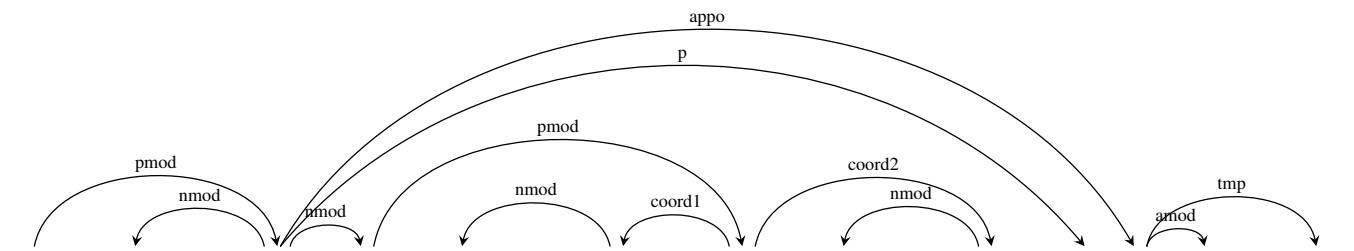

... September reports on industrial production and capacity utilization, also due tomorrow

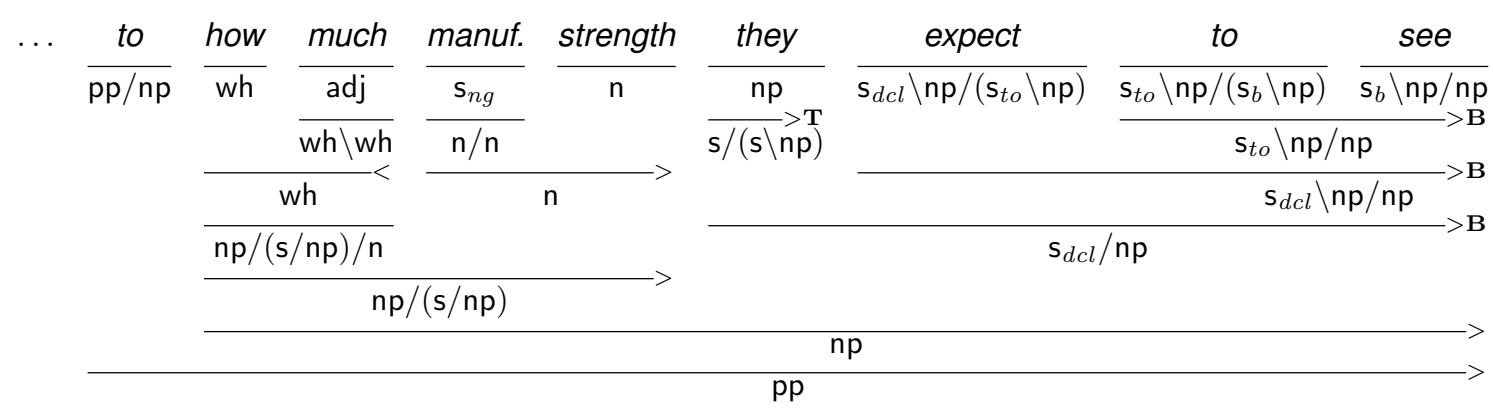

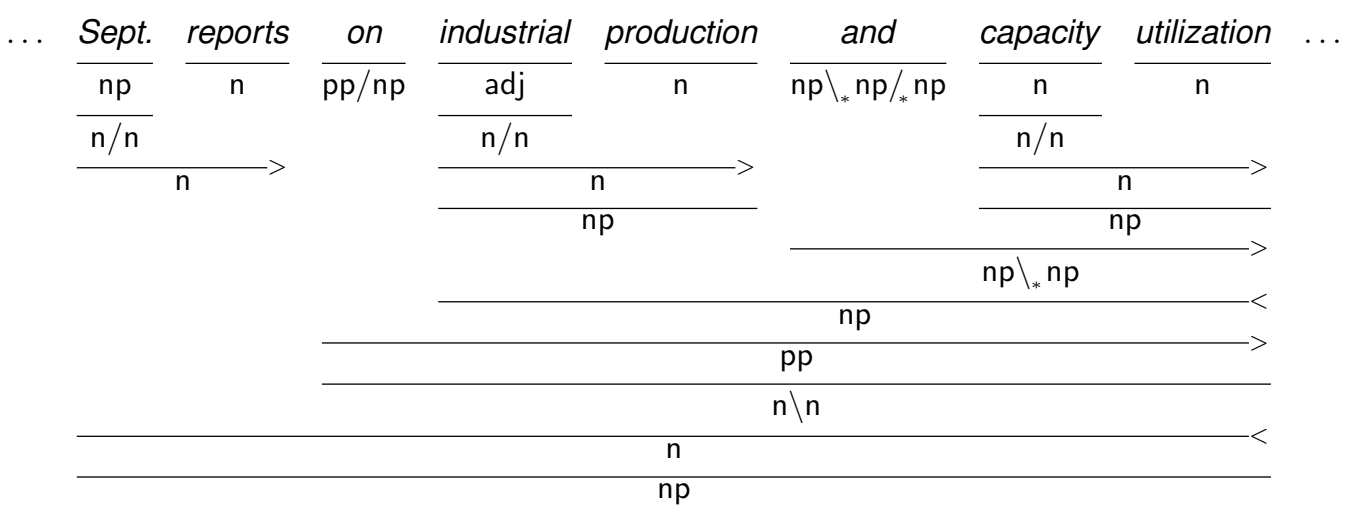

Figure 2: Augmented Syntactic Dependencies with Corresponding CCG Derivation (dashed dependencies indicate relations from additional parents beyond those in the primary tree structure) 
(3) expect $\vdash \mathbf{s}_{w_{10}, \mathrm{dcl}} \backslash \mathrm{np}_{w_{9}} /\left(\mathbf{s}_{w_{11}, \text { to }} \backslash \mathrm{np}_{w_{9}}\right)$ :

$@_{w_{10}}($ expect $\wedge\langle$ TENSE $\rangle$ pres $\wedge$

$\langle$ SUBJ $\rangle w_{9} \wedge\langle$ OPRED $\left.\rangle w_{11}\right)$

(4) and $\vdash \mathrm{np}_{w_{19}} \backslash_{*} \mathrm{np}_{w_{18}} /_{*} \mathrm{np}_{w_{21}}$ :

$@_{w_{19}}\left(\right.$ and $\wedge\langle$ COORD 1$\rangle w_{18} \wedge\langle$ COORD 2$\left.\rangle w_{21}\right)$

(5) $\mathrm{n}_{w_{20}} \Rightarrow \mathrm{n}_{w_{21}} / \mathrm{n}_{w_{21}}: @_{w_{21}}\left(\langle\mathrm{NMOD}\rangle w_{20}\right)$

At the end of the recursion, the lexical categories and type-changing rules are extracted from the highest-scoring derivation and added to the output sets, after first replacing indices such as $w_{10}$ with variables.

\section{Experiments and Future Work}

We ran the induction algorithm over the standard PTB training sections (02-21), recovering complete derivations more than $90 \%$ of the time for most sections. Robust treatment of coordination, including argument cluster coordination and gapping, remains a known issue; other causes of derivation failures remain to be investigated. To select preferred derivations, we used a complexity metric that simply counts the number of steps and the number of slashes in the categories. We then trained a generative syntactic model (Hockenmaier and Steedman, 2002) and used it along with a composite language model to generate $n$ best realizations for reranking (White and Rajkumar, 2012), additionally using a large-scale (gigaword) language model. Development and test results appear in Table 1. Perhaps because of the expanded use of type-changing rules with simple lexical categories, the generative model and hypertagger (Espinosa et al., 2008) performed worse than expected. Combining the generative syntactic model and composite language model (GEN) with equal weight yielded a devtest BLEU score of only 0.4513 , while discriminatively training the generative component models (GLOBAL) increased the score to 0.7679 . Using all features increased the score to 0.8083 , while doubling the beam size (ALL+) pushed the score to 0.8210 , indicating that search errors may be an issue. Ablation results show that leaving out the large-scale language model (NO-BIGLM) and dependency-ordering features (NO-DEPORD) substantially drops the score. ${ }^{3}$ Focusing only on the $80.5 \%$ of the sentences for which a complete derivation was found (COMPLETE) yielded a score of 0.8668. By comparison, realization with the

\footnotetext{
${ }^{3}$ All differences were statistically significant at $p<0.01$ with paired bootstrap resampling (Koehn, 2004).
}

\begin{tabular}{lrcc}
\hline Model & Exact & Complete & BLEU \\
\hline Sect 00 & & & \\
GEN & 2.4 & 79.5 & 0.4513 \\
GLOBAL & 29.7 & 79.0 & 0.7679 \\
\hline NO-BIGLM & 29.1 & 78.2 & 0.7757 \\
NO-DEPORD & 34.3 & 77.9 & 0.7956 \\
ALL & 35.8 & 78.4 & 0.8083 \\
ALL+ & 36.4 & 80.5 & $\mathbf{0 . 8 2 1 0}$ \\
COMPLETE & 44.4 & - & 0.8668 \\
\hline NATIVE & 48.0 & 88.7 & 0.8793 \\
& & & \\
\hline Sect 23 & & & \\
GEN & 2.8 & 80.3 & 0.4560 \\
GLOBAL & 31.3 & 78.5 & 0.7675 \\
ALL & 37.6 & 77.2 & 0.8083 \\
ALL+ & 38.1 & 80.4 & $\mathbf{0 . 8 2 6 0}$ \\
COMPLETE & 47.0 & - & 0.8743 \\
\hline NATIVE & 46.4 & 86.4 & 0.8694
\end{tabular}

Table 1: Development set (Section 00) \& test set (Section 23) results, including exact match and complete derivation percentages and BLEU scores

native OpenCCG inputs (and the large-scale LM) on all sentences (NATIVE) yields a score more than five BLEU points higher, despite using inputs with more semantically-oriented relations and leaving out many function words, indicating that there is likely substantial room for improvement in the pre-processing and grammar induction process. Towards that end, we tried selecting the best derivations using several rounds of Viterbi EM with the generative syntactic model, but doing so did not improve realization quality.

A similar pattern is seen in the Section 23 results, with a competitive BLEU score of 0.8260 with the expanded beam, much higher than Narayan \& Gardent's (2012) score of 0.675 with $38.8 \%$ coverage, the best previous score with a grammar-based system. This score still trails the shared task scores of the top statistical dependency realizers by several points (STUMABA-S at 0.8911 and DCU at 0.8575), though it exceeds the score of a purpose-built system using no external resources (ATT at 0.6701). In future work, we hope to close the gap with the top systems by integrating an improved ranking model into the induction process and resolving the remaining representational issues with problematic constructions.

\section{Acknowledgments}

Thanks to the anonymous reviewers, Richard Johansson and the University of Sydney Schwa Lab for helpful comments and discussion. This work was supported in part by NSF grants IIS-1143635 and IIS-1319318. 


\section{References}

Bharat Ram Ambati, Tejaswini Deoskar, and Mark Steedman. 2013. Using CCG categories to improve Hindi dependency parsing. In Proc. ACL.

Yoav Artzi and Luke Zettlemoyer. 2013. Weakly supervised learning of semantic parsers for mapping instructions to actions. TACL, 1:49-62.

Anja Belz, Michael White, Dominic Espinosa, Eric Kow, Deirdre Hogan, and Amanda Stent. 2011. The first surface realisation shared task: Overview and evaluation results. In Proc. ENLG.

Dominic Espinosa, Michael White, and Dennis Mehay. 2008. Hypertagging: Supertagging for surface realization with CCG. In Proc. ACL.

Julia Hockenmaier and Mark Steedman. 2002. Generative models for statistical parsing with Combinatory Categorial Grammar. In Proc. ACL.

Philipp Koehn. 2004. Statistical significance tests for machine translation evaluation. In Proc. EMNLP.

Tom Kwiatkowksi, Luke Zettlemoyer, Sharon Goldwater, and Mark Steedman. 2010. Inducing probabilistic CCG grammars from logical form with higherorder unification. In Proc. EMNLP.

Shashi Narayan and Claire Gardent. 2012. Error mining with suspicion trees: Seeing the forest for the trees. In Proc. COLING.

Michael White and Rajakrishnan Rajkumar. 2012. Minimal dependency length in realization ranking. In Proc. EMNLP. 